\title{
Veverimer for treatment of chronic metabolic acidosis in patients with chronic kidney disease
}

\author{
Anirudh R. Gone ${ }^{1}$, Wei Chen ${ }^{1,2}$ \\ ${ }^{1}$ Department of Medicine, Montefiore Medical Center, Albert Einstein College of Medicine, Bronx, NY, USA; ${ }^{2}$ Department of Medicine, University \\ of Rochester School of Medicine and Dentistry, Rochester, NY, USA \\ Correspondence to: Wei Chen, MD, MS. Assistant Professor of Medicine, Albert Einstein College of Medicine, 1300 Morris Park Avenue, Ullmann \\ 615, Bronx, NY 10461, USA. Email: weichen@montefiore.org. \\ Comment on: Wesson DE, Mathur V, Tangri N, et al. Long-term safety and efficacy of veverimer in patients with metabolic acidosis in chronic kidney \\ disease: a multicentre, randomised, blinded, placebo-controlled, 40-week extension. Lancet 2019;394:396-406.
}

Submitted Apr 14, 2020. Accepted for publication May 09, 2020.

doi: $10.21037 /$ atm-20-3320

View this article at: http://dx.doi.org/10.21037/atm-20-3320

\section{Chronic metabolic acidosis in chronic kidney disease}

Due to population growth and aging, the burden of chronic kidney disease (CKD) increased substantially worldwide. According to an epidemiology study conducted using the Global Burden of Disease study data, the prevalence of CKD increased by $87 \%$ and death due to CKD increased by $98 \%$ in the past 3 decades (1). Kidneys are essential in maintaining fluid and electrolyte balance in the body, and one of the major functions of kidneys is the regulation of acid-base balance. Compared to the ancestral human diets that are rich in plants, contemporary diets are rich in animal proteins, which are metabolized to acids. Kidneys excrete excess acid by reabsorbing filtered bicarbonate and generating new bicarbonate (2). The ability of the kidneys to excrete acid decreases as kidney function worsens; this results in acid retention and chronic metabolic acidosis (2). It is estimated that $\sim 15 \%$ of patients with CKD have chronic metabolic acidosis (3).

Chronic metabolic acidosis is associated with several adverse consequences such as CKD progression, muscle wasting, bone loss, and cardiovascular disease (4). Clinical practice guidelines suggest to treat chronic metabolic acidosis with alkali therapy to neutralize the accumulated acid $(5,6)$. In patients with CKD, alkali therapy, which includes mainly sodium bicarbonate and base-producing fruits and vegetables, has been shown not only to improve acid-base status, but also may preserve kidney function, improve muscle mass, and vascular endothelial function (7).
However, the evidence supporting these beneficial effects of alkali therapy is limited by suboptimal quality of existing clinical studies, such as small sample size and lack of blinding for participants (8). In addition, sodium bicarbonate supplementation may lead to sodium retention limiting the use of sodium bicarbonate in patients with heart failure and hypertension, which are conditions that often co-exist with CKD (9). Dietary intervention with fruits and vegetables is difficult to implement, and consumption of base-producing fruits and vegetables may raise serum potassium levels, which may already be high in patients with CKD.

\section{Emergence of veverimer for treating chronic metabolic acidosis}

Veverimer is a non-absorbable hydrochloric acid binder. After ingestion, veverimer is protonated and binds to chloride anions, thus reducing the amount of hydrochloric acid in the gastrointestinal tract (Figure 1) (10). It is nonabsorbable; and compared to sodium bicarbonate, it does not lead to sodium retention. This editorial discusses a recent study that investigated the long-term safety and efficacy of veverimer, published in The Lancet by Wesson et al., entitled, "Long-term safety and efficacy of veverimer in patients with metabolic acidosis in chronic kidney disease: a multicentre, randomised, blinded, placebo-controlled, 40-week extension" (11).

Prior to the featured article, the group published 2 other 


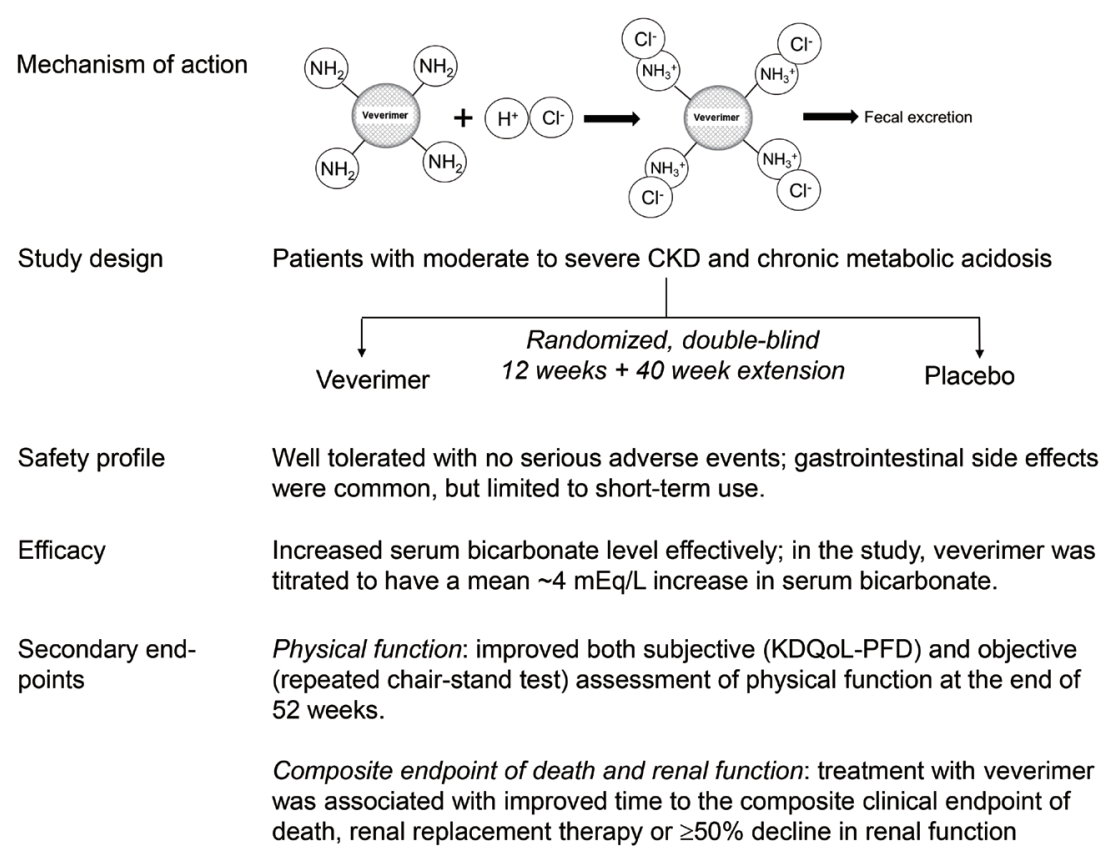

Figure 1 Summary of the veverimer studies to treat chronic metabolic acidosis in CKD. Veverimer is a nonabsorbable hydrochloric acid binder. After ingestion, veverimer is protonated and binds to chloride anions, thus reducing the amount of hydrochloric acid in the gastrointestinal tract. To study the efficacy and safety of veverimer, 217 patients with moderate to severe CKD (eGFR between 20 and $40 \mathrm{~mL} / \mathrm{min}$ per $1.73 \mathrm{~m}^{2}$ ) and serum bicarbonate concentration between 12 and $20 \mathrm{mEq} / \mathrm{L}$ were randomized to receive either veverimer or placebo for 12 weeks; then 196 continued on their blinded treatment assignment for another 40 weeks. Veverimer was well tolerated with no serious adverse events, except short-term gastrointestinal side effects such as diarrhea. Compared to placebo, veverimer effectively increased serum bicarbonate level. Serum bicarbonate levels started to increase after 1 week of veverimer. At week 4, serum bicarbonate increased by $\sim 4 \mathrm{mEq} / \mathrm{L}$ in average and this increase was sustained for the remaining 48 weeks of veverimer treatment. Veverimer improved physical function after 52 weeks, which were measured subjectively measured by KDQoL-PFD survey and objectively by repeated chairstand test. Lastly, treatment with veverimer was associated with improved time to the composite clinical endpoint of death, initiation of renal replacement therapy or having $\geq 50 \%$ reduction in eGFR. CKD, chronic kidney disease; KDQoL-PFD, physical function domain of Kidney Disease and Quality of Life; eGFR, estimated glomerular filtrate rate.

studies on veverimer. The first was a 2 -week phase $1 / 2$ study, in which, 135 participants with moderate to severe CKD (estimated glomerular filtrate rate (eGFR) between 20 and $<60 \mathrm{~mL} / \mathrm{min}$ per $1.73 \mathrm{~m}^{2}$ ) and serum bicarbonate level between 12 and $20 \mathrm{mEq} / \mathrm{L}$ were randomized to receive either veverimer or placebo (12). There are many ways to assess acid-base balance in CKD, and they include arterial blood gas, serum bicarbonate level, and net acid balance, which is the difference between dietary acid load and renal acid excretion. Clinically, serum bicarbonate level is most often used to diagnose and guide the treatment of chronic metabolic acidosis in patients with CKD. Guidelines recommend to use alkali therapy to treat chronic metabolic acidosis and to maintain a serum bicarbonate level $\geq 22 \mathrm{mEq} / \mathrm{L}$ (5). By the end of the phase $1 / 2$ study, serum bicarbonate level did not change in the placebo group, but increased by a mean of 3.2 to $3.9 \mathrm{mEq} / \mathrm{L}$ in the treatment group (12).

The other study was a 12 -week randomized, doubleblind, placebo-controlled phase 3 trial (10). The study was conducted at 37 sites in 8 countries. A total of 217 patients with eGFR between 20 and $40 \mathrm{~mL} / \mathrm{min}$ per $1.73 \mathrm{~m}^{2}$ and serum bicarbonate concentration between 12 and $20 \mathrm{mEq} / \mathrm{L}$ were randomized to receive either veverimer $(n=124)$ or placebo $(n=93)$ once daily. Some of the key characteristics of the study participants are shown in Table 1. The primary efficacy endpoint was a composite of the difference between the treatment and placebo groups in the proportion of patients achieving either an increase of $\geq 4 \mathrm{mEq} / \mathrm{L}$ in serum bicarbonate level from the baseline or achieving a serum 
Table 1 Characteristics of study participants and drug information on veverimer*

\begin{tabular}{|c|c|}
\hline Variables & Outcomes \\
\hline Age (years) & $\sim 63 \pm 12$ \\
\hline eGFR $\left(\mathrm{mL} / \mathrm{min} / 1.73 \mathrm{~m}^{2}\right)$ & $\sim 28 \pm 6$ \\
\hline Serum bicarbonate (mEq/L) & $17 \pm 1.5$ \\
\hline Hypertension $^{\mathrm{b}}(\%)$ & $97 \%$ \\
\hline Congestive heart failure $^{c}(\%)$ & $29-33 \%$ \\
\hline \multicolumn{2}{|c|}{ Drug information based on veverimer research studies (not be used in clinical setting) } \\
\hline Dose studied & 6 gram per day \\
\hline Mechanism of action & Binds to hydrochloric acid in the intestinal tract \\
\hline Onset of action & Increase in serum bicarbonate within the first $24-48$ hours \\
\hline Excretion & Fecal \\
\hline Common adverse effect & Gastrointestinal, most commonly diarrhea \\
\hline Monitor & Serum bicarbonate \\
\hline
\end{tabular}

Age, eGFR and serum bicarbonate are shown as mean \pm standard deviation. Diabetes, hypertension and congestive heart failure are shown in percentage. *, table generated based on the available information published in the veverimer studies (10-12); ${ }^{\text {a }}$, participants with glycated hemoglobin A1C >9\% were excluded; ${ }^{b}$, participants with systolic blood pressure $\geq 170 \mathrm{mmHg}$ were excluded; ${ }^{c}$, participants with New York Heart Association Class IV symptoms were excluded. eGFR, estimated glomerular filtration rate.

bicarbonate to the normal range of 22 to $29 \mathrm{mEq} / \mathrm{L}$. After 12 weeks, $59 \%$ of participants in the veverimer group met the primary endpoint, significantly higher than the placebo group (22\%, $\mathrm{p}<0.001)$. Similar to the phase $1 / 2$ study (12), serum bicarbonate increased by a mean of $4.4 \mathrm{mEq} / \mathrm{L}$ in the veverimer group compared to $1.8 \mathrm{mEq} / \mathrm{L}$ in the placebo group $(\mathrm{P}<0.001)$. The most common side effect of veverimer was in the gastrointestinal system, especially diarrhea, and no serious adverse effects was observed. Both phase $1 / 2$ and phase 3 studies were limited by short term duration of follow up.

\section{Long-term safety and efficacy of veverimer}

To evaluate the long-term safety and efficacy of veverimer, participants from the phase 3 trial was followed for an additional 40 weeks and the findings were reported in the featured article $(10,11)$. Namely, the featured study is an extension of the parent phase 3 trial; and the total study duration was 52 weeks or 1 year. Of the 217 patients randomized in the parent study, 196 patients continued on their blinded treatment assignment (114 veverimer and 82 placebo) (11). Veverimer had a similar safety profile with placebo after 52 weeks of follow up. In the parent phase 3 study, the most common adverse effects were in the gastrointestinal system (13\% in patients from the treatment group versus $5 \%$ in the placebo group) (10). However, in the extension study, more participants in the placebo group (26\%) had gastrointestinal adverse events than in the treatment group (21\%). No participants on veverimer discontinued the medication due to an adverse event. Serious adverse events occurred in $2 \%$ of participants in the treatment group and 5\% in the placebo group, and none of these events were judged to be related to veverimer. We summarized some of the drug information on veverimer in Table 1.

In the parent study (10), more participants in the treatment group than the placebo group met the primary efficacy endpoint of raising serum bicarbonate level. Similar results were seen after 52 weeks: $63 \%$ participants 
in the treatment group compared to $38 \%$ in the placebo group $(\mathrm{P}=0.0015)$ met the primary endpoint (11). Serum bicarbonate levels started to increase after 1 week of veverimer. By 4 weeks, serum bicarbonate increased by $\sim 4 \mathrm{mEq} / \mathrm{L}$ in average and this increase was sustained for the remaining 48 weeks while on veverimer. After 52 weeks, both veverimer and placebo were discontinued; and after 2 weeks off veverimer, serum bicarbonate in the treatment group dropped to a level similar to that in the placebo group. The studies demonstrated that veverimer was effective in increasing serum bicarbonate levels in patients with CKD and chronic metabolic acidosis; while gastrointestinal side effects may occur, long-term use of veverimer for up to 1 year seems to be safe.

\section{Effect of veverimer on physical function}

Both parent and the extension study further explored the effect of veverimer on physical function as their secondary endpoints. Patients with CKD have reduced physical function, which is associated with morbidity and mortality (13). Acidosis has been linked with skeletal muscle protein metabolism. Acidosis may contribute to muscle wasting by impairing insulin/insulin-like growth factor-1 signaling and stimulating proteolysis (14). Some studies have shown that sodium bicarbonate therapy in patients with CKD increased muscle mass and improved physical function $(15,16)$, but many of these studies had small sample size or were conducted without blinding the participants. Two doubleblind placebo-controlled trials of sodium bicarbonate therapy on physical function were published recently and both showed no improvement in physical function $(17,18)$. The effect of sodium bicarbonate therapy on physical function in patients with CKD remains controversial.

In the veverimer studies $(10,11)$, physical function was assessed both subjectively and objectively. Subjectively, participants were asked to complete the physical function domain of Kidney Disease and Quality of Life (KDQoLPFD) survey at baseline, week 12, 40, and 50. The KDQoLPFD survey quantifies patients' self-reported degree of limitation in doing daily activities such as climbing stairs and walking, and the score ranges from 0 to 100 points with a higher score indicating better physical function. At the end of 12 weeks, KDQoL-PFD scores increased significantly in the treatment group compared with placebo by approximately 5 points. This increase was sustained until week 40. At week 52, the scores increased by another 6 points on average in the treatment group, whereas the scores in the placebo group decreased $\sim 1$ point compared to baseline.

Objectively, physical function was measured by repeated chair-stand tests, which measure the time to complete repeated standing from a chair 5 times as rapidly as possible. A shorter time needed to complete the task or a faster speed reflects better physical function. At the end of 12 weeks, there was no statistically significant improvement in the performance of repeated chair-stand tests in the treatment group, but the $\mathrm{P}$ value was close to reaching a statistical significance $(\mathrm{P}=0.06)$. By week 40 and 52, an improvement in the speed of chair-stand tests became apparent. Compared to baseline, the time required to complete the chair-stand test was reduced by $\sim 4$ seconds in the treatment group, whereas there was only 1.5 second reduction in placebo. This improvement was both statistically significant $(\mathrm{P}<0.001)$ and clinically meaningful. Thus, the study demonstrated that treatment with veverimer for 1 year improved physical function both subjectively and objectively.

To date, this is the best evidence to support that correcting chronic metabolic acidosis improves physical function in patients with CKD. As mentioned earlier, two recently published, double-blind, placebo-controlled trials showed that sodium bicarbonate therapy for 2 years did not improve physical function $(17,18)$. One of the studies was conducted in the United States (US) (17), and the other was in the United Kingdom (UK) (18). Compared to the veverimer studies, participants in the US had higher kidney function (mean eGFR was 36 versus $\sim 29 \mathrm{~mL} / \mathrm{min} / 1.73 \mathrm{~m}^{2}$ in the veverimer studies) and milder acidosis (mean serum bicarbonate level was 24 versus $17 \mathrm{mEq} / \mathrm{L}$ in the veverimer studies) at baseline. In the UK, the participants also had higher serum bicarbonate levels at baseline (mean $\sim 20 \mathrm{mEq} / \mathrm{L}$ ), and the study population were much older (mean age 74 versus 63 years in veverimer studies). The differences in the study population may partly explain the differences between the results of sodium bicarbonate trials and veverimer studies, or perhaps, the findings from the veverimer studies cannot be extrapolated to the use of sodium bicarbonate.

\section{Veverimer and CKD progression}

The effect of veverimer on kidney function was also explored as a pre-specified endpoint. Multiple epidemiologic studies have demonstrated that lower serum bicarbonate concentrations were associated with faster CKD progression (17), and lower kidney function was 
associated with higher mortality in CKD. In a US cohort of CKD patients (18), for every $10 \mathrm{~mL} / \mathrm{min} / 1.73 \mathrm{~m}^{2}$ lower in eGFR, the adjusted relative risk of death was 1.28 (95\% confidence interval: 1.12 to 1.45$)$. Metabolic acidosis may promote tubulointerstitial injury in the kidneys by activating ammonia-induced complement and by increasing the production of endothelin-1 and aldosterone (14); this in turn contributes to CKD progression. Several interventional studies have shown that alkali therapy using either sodium bicarbonate or fruits and vegetables may preserve kidney function (8). For example, in a study of 188 patients with moderate to severe CKD and serum bicarbonate level $<22 \mathrm{mEq} / \mathrm{L}$, less participants in the sodium bicarbonate group had a rapid decline in kidney function compared to the control group without sodium bicarbonate $(20 \% v s$. $40 \%, \mathrm{P}=0.001$ ) in 6 months (16). The veverimer extension study was under-powered to evaluate the effects of veverimer on CKD progression (11); however, compared to placebo, participants in the treatment group were less like to die, initiate renal replacement therapy or have a at least $50 \%$ decline in kidney function during the 1 year follow up period (incidence rate was $4 \%$ in treatment group $v$ s. $12 \%$ in placebo, $\mathrm{P}=0.02$ ).

\section{Discussion and future studies}

In summary, veverimer, a novel hydrochloric acid binder, has been shown to effectively increase serum bicarbonate level in patients with CKD and chronic metabolic acidosis. In this 1-year study, veverimer was well tolerated and had a decent safety profile, except for short-term gastrointestinal side effects. Treatment with veverimer improved physical function and was associated with better outcomes in mortality and renal prognosis. Compared to the published clinical trials on sodium bicarbonate therapy (17), the veverimer studies have several strengths, including being an international study and having a relatively low risk of study biases. The studies were well designed and carefully executed. There are few limitations. First, it was probably difficult to maintain blinding throughout the entire study. Since the phase $1 / 2$ study had already shown the efficacy of veverimer in improving serum bicarbonate level (12), checking serum bicarbonate per study protocol might have caused the loss of blinding, at least for the investigators, during the study. This could have generated bias favoring veverimer in terms of clinical outcomes such as physical function. Second, patients with systolic blood pressure $\geq 170$ $\mathrm{mmHg}$ and advanced heart failure were excluded from the veverimer studies. Inclusion of these patients could have better demonstrated the superiority of veverimer over sodium bicarbonate, which may lead to sodium retention.

Overall, veverimer is a promising therapeutic option for treating chronic metabolic acidosis, especially in patients who have contraindications to receive sodium bicarbonate. The application of veverimer to seek the approval from Food and Drug Administration (FDA) in the US is in progress (19). A major foreseeable challenge of using veverimer is its cost. Sodium bicarbonate pills are relatively inexpensive. Estimated monthly cost of sodium bicarbonate is approximately 3 USD (US dollar), using $1.0 \mathrm{mEq} / \mathrm{kg}$ per day as the recommended dose and assuming $70 \mathrm{~kg}$ as the body weight (20-22). The price of veverimer is yet to be determined; however, the cost of recently FDA approved oral binders, such as patiromer, a potassium binder, range from 600 to 800 USD per month (23).

Meanwhile, a phase $3 \mathrm{~b}$ study of veverimer is currently ongoing. The phase $3 \mathrm{~b}$ study is a randomized, double-blind, placebo-controlled trial, in which 1,600 participants are randomized to receive veverimer or placebo (24). The study population is similar to the phase 3 study and participants will have an average of 3.5-year follow up. The primary endpoint is the progression of CKD, and the secondary endpoints include time to end stage renal disease, physical function, cardiovascular mortality and hospitalization. The study is expected to complete by November 2022. The study of this magnitude has never been conducted in the field of chronic metabolic acidosis. The findings from this phase $3 \mathrm{~b}$ study will certainly make a significant contribution and advance the science in the field.

\section{Acknowledgments}

Funding: WC is supported by K23 DK114476 from the National Institutes of Health and Carl W. Gottschalk Research Scholar Grant from the American Society of Nephrology.

\section{Footnote}

Provenance and Peer Review: This article was commissioned and reviewed by the Section Editor Dr. Cheng Yuan (Zhongnan Hospital, Wuhan University, China).

Conflicts of Interest: Both authors have completed the ICMJE uniform disclosure form (available at http://dx.doi. org/10.21037/atm-20-3320). The authors have no conflicts 
of interest to declare.

Ethical Statement: All authors are accountable for all aspect of the work in ensuring that questions related to the accuracy or integrity of any part of the work are appropriately investigated and resolved.

Open Access Statement: This is an Open Access article distributed in accordance with the Creative Commons Attribution-NonCommercial-NoDerivs 4.0 International License (CC BY-NC-ND 4.0), which permits the noncommercial replication and distribution of the article with the strict proviso that no changes or edits are made and the original work is properly cited (including links to both the formal publication through the relevant DOI and the license). See: https://creativecommons.org/licenses/by-nc-nd/4.0/.

\section{References}

1. Xie Y, Bowe B, Mokdad AH, et al. Analysis of the Global Burden of Disease study highlights the global, regional, and national trends of chronic kidney disease epidemiology from 1990 to 2016. Kidney Int 2018;94:567-81.

2. Nagami GT, Hamm LL. Regulation of Acid-Base Balance in Chronic Kidney Disease. Adv Chronic Kidney Dis 2017;24:274-9.

3. Chen W, Abramowitz MK. Epidemiology of AcidBase Derangements in CKD. Adv Chronic Kidney Dis 2017;24:280-8.

4. Kraut JA, Madias NE. Metabolic Acidosis of CKD: An Update. Am J Kidney Dis 2016;67:307-17.

5. National Kidney F. K/DOQI clinical practice guidelines for chronic kidney disease: evaluation, classification, and stratification. Am J Kidney Dis 2002;39:S1-266.

6. Inker LA, Astor BC, Fox CH, et al. KDOQI US commentary on the 2012 KDIGO clinical practice guideline for the evaluation and management of CKD. Am J Kidney Dis 2014;63:713-35.

7. Chen W, Abramowitz MK. Advances in management of chronic metabolic acidosis in chronic kidney disease. Curr Opin Nephrol Hypertens 2019;28:409-16.

8. Chen W, Levy DS, Abramowitz MK. Acid Base Balance and Progression of Kidney Disease. Semin Nephrol 2019;39:406-17.

9. Bushinsky DA. Tolerance to Sodium in Patients With CKD-Induced Metabolic Acidosis: Does the Accompanying Anion Matter? Am J Kidney Dis 2019;73:858-65.
10. Wesson DE, Mathur V, Tangri N, et al. Veverimer versus placebo in patients with metabolic acidosis associated with chronic kidney disease: a multicentre, randomised, double-blind, controlled, phase 3 trial. Lancet 2019;393:1417-27.

11. Wesson DE, Mathur V, Tangri N, et al. Long-term safety and efficacy of veverimer in patients with metabolic acidosis in chronic kidney disease: a multicentre, randomised, blinded, placebo-controlled, 40-week extension. Lancet 2019;394:396-406.

12. Bushinsky DA, Hostetter T, Klaerner G, et al. Randomized, Controlled Trial of TRC101 to Increase Serum Bicarbonate in Patients with CKD. Clin J Am Soc Nephrol 2018;13:26-35.

13. Weiner DE, Seliger SL. Cognitive and physical function in chronic kidney disease. Curr Opin Nephrol Hypertens 2014;23:291-7.

14. Chen W, Abramowitz MK. Treatment of metabolic acidosis in patients with CKD. Am J Kidney Dis 2014;63:311-7.

15. Abramowitz MK, Melamed ML, Bauer C, et al. Effects of oral sodium bicarbonate in patients with CKD. Clin J Am Soc Nephrol 2013;8:714-20.

16. Dubey AK, Sahoo J, Vairappan B, et al. Correction of metabolic acidosis improves muscle mass and renal function in chronic kidney disease stages 3 and 4: a randomized controlled trial. Nephrol Dial Transplant 2020;35:121-9.

17. Chen W, Levy D, Abramowitz M. Acid Base Balance and Progression of Kidney Disease. Semin Nephrol 2019;39:406-17.

18. Kovesdy CP, Trivedi BK, Anderson JE. Association of kidney function with mortality in patients with chronic kidney disease not yet on dialysis: a historical prospective cohort study. Adv Chronic Kidney Dis 2006;13:183-8.

19. Development Status and FDA Approval Process for veverimer. Retrieved April 14, 2020. Available online: https://www.drugs.com/history/veverimer.html.

20. Mathur RP, Dash SC, Gupta N, et al. Effects of correction of metabolic acidosis on blood urea and bone metabolism in patients with mild to moderate chronic kidney disease: a prospective randomized single blind controlled trial. Ren Fail 2006;28:1-5.

21. Bellasi A, Di Micco L, Santoro D, et al. Correction of metabolic acidosis improves insulin resistance in chronic kidney disease. BMC Nephrol 2016;17:158.

22. Sodium bicarbonate Prices, Coupons and Patient Assistance Programs. Retrieved April 27, 2020. Available 
online: https://www.drugs.com/price-guide/sodiumbicarbonate.

23. Veltassa. Retrieved April 27, 2020. Available online: https://www.goodrx.com/veltassa

24. Tricida (2019, February 26, 2019). Evaluation of Effect

Cite this article as: Gone AR, Chen W. Veverimer for treatment of chronic metabolic acidosis in patients with chronic kidney disease. Ann Transl Med 2020;8(22):1539. doi: 10.21037/ atm-20-3320 of TRC101 on Progression of Chronic Kidney Disease in Subjects With Metabolic Acidosis (VALOR-CKD). Retrieved March 14, 2019. Available online: https:// clinicaltrials.gov/ct2/show/NCT03710291 